\title{
A Dynamic Bayesian Network for Handling Uncertainty in a Decision Support System Adapted to the Monitoring of Patients Treated by Hemodialysis
}

\author{
Cédric Rose, Cherif Smaili, and François Charpillet \\ INRIA-LORIA, B.P.239 \\ 54506 Vandoeuvre-lès-Nancy, France \\ \{rose,smailic,charpillet\}@loria.fr
}

\begin{abstract}
Telemedicine is a mean of facilitating the distribution of human resources and professional competences. It can speed up diagnosis and therapeutic care delivery and allow peripheral healthcare providers to receive continuous assistance from specialized centers. The need of specialized human resources becomes critical with the aging of the population. The treatment of renal failure is an example where telemedicine can help to increase care quality. Over the last decades Bayesian networks has become a popular representation for encoding uncertain expert knowledge. Dynamic Bayesian networks are an extension of Bayesian networks for modeling dynamic processes. We developed a dynamic Bayesian network adapted to the monitoring of the dry weight of patients suffering from chronic renal failure treated by hemodialysis. An experimentation conducted at dialysis units indicated that the system is reliable and gets the approbation of its users.
\end{abstract}

\section{Introduction}

Telemedicine is the use of telecommunication technologies for medical diagnosis and patient care. It can deliver health care services when the provider and the client are separated by distance. Health care professionals can now access or exchange information for diagnosis, treatment and prevention of diseases and injuries [7]. The medical action depends on the doctors reasoning capacity and his aptitude to make decisions relying on potentially uncertain information. This uncertainty has multiple origins: data errors, ambiguity of the representation of information, uncertainty about the relations between various source of information...

A first approach to deal with the problem of knowledge representation in an uncertainty context is to use probability theory. Bayesian networks have become a popular representation in Artificial Intelligence for encoding uncertain knowledge [8] [9]. In parallel to the progress on the theoretical foundation of knowledge representation, advances have also been made in the algorithmic development of efficient inference schemes [1] [3] [2].

In this article, we develop a decision support system for surveillance of patients suffering from renal failure and treated by hemodialysis. The next section is a brief introduction to Bayesian networks and dynamic Bayesian networks. In section 3 , we present the medical problem in which we are interested. Section 4 describes the network model for diagnosis and decision making. Section 5 and 6 present the different stages of the medical data analysis and the generation of alerts. Finally, we evaluate the potential of our approach by presenting some experiments and results.

\section{Bayesian Networks}

Graphical models are a marriage between probability theory and graph theory. They provide a natural tool for dealing with two problems that occur throughout applied mathematics and engineering: uncertainty and complexity [6]. Particularly they are playing an increasingly important role in many medical applications due to their capacity to represent uncertain knowledge.

A Bayesian network (also known as Belief Network or Directed Probabilistic Independence Network) [5] [4] is a graph with probabilities for representing random variables and their dependencies. It efficiently encodes the joint probability distribution of a set of variables. Its nodes represent random variables and its arcs represent dependencies between the random variables encoded by conditional probabilities.

Dynamic Bayesian networks (DBN) [6] encode the joint probability distribution of a time-evolving set of variables $X[t]=\left\{X_{1}[t], \ldots, X_{N}[t]\right\}$. For a given time interval of size $T$, a dynamic Bayesian network can be considered as a 'static' Bayesian network with $T \times N$ variables. DBN are a generalization of Kalman Filter Models (KFM) and Hidden 
Markov Models (HMM) [10].

\section{Application context}

The human body gains water by drinking, eating but it also has a metabolic production of water. The water is eliminated by respiration, defecation, sweating and urination, which is the only regulated loss. The kidney is responsible for keeping constant the amount of water as needed by the organism. The progressive or sudden loss of this ability is known as kidney failure. Chronic kidney failure treatments are renal dialysis and kidney transplant.

By doing dialysis, the nephrologist intends to maintain his patient in a normally hydrated state which corresponds to a reference body weight for the patient known as the dry weight. A major difficulty of the treatment is the estimation of this ideal weight since the hydration level of the patient cannot be directly measured. The evaluation made by the physician is mainly based on the monitoring of the body weight and the blood pressure of the patient. As shown on figure 1, a misadjusted dry weight has the consequence to overhydrate or a dehydrate the patient.

DialHemo is a national INRIA action for developing a tele-surveillance and tele-diagnosis system adapted to kidney failure patients treated by hemodialysis either at home or at autodialysis units. The system will be accessible by nephrologists as well as general practitioners. Several dialysis units are now connected to the system.

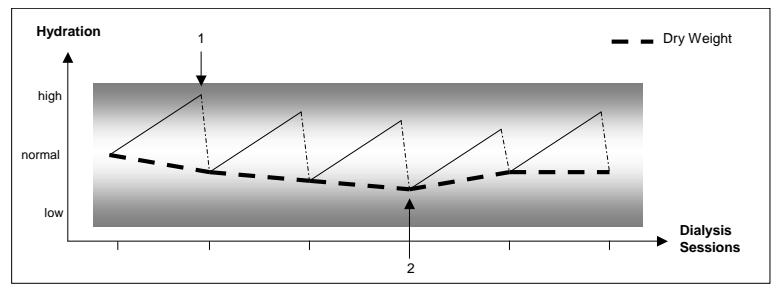

Figure 1. On this figure we see that the patient gains water between dialysis sessions and loose water during the dialysis sessions. The estimation of the dry weight is made empirically. When the patient presents signs of overhydration (1) the dry weight should be decreased. If the patient is dehydrated at the end of the dialysis session (2) the dry weight has to be increased.

The monitoring of the dry weight adjustment in hemodialysis is a regulation problem. The patient provides information on his hydration state (weight, blood pressure,...) and, by his actions (increase/decrease the dry weight), the physician modifies the hydration state of his patient. The decision support system can be considered as a third agent interacting with both the patient and the physician (see figure 2). It improves the communication of information from the patient to the physician by processing the data and selecting the information of interest. The decision support system does not replace the physician visits. It comes in addition, providing information between the visits. The processing and selection of information in a medical context is certainly a delicate matter. The information provided should be relevant. The system must not miss real alerts and, on the other hand, it should not produce to much false alerts.

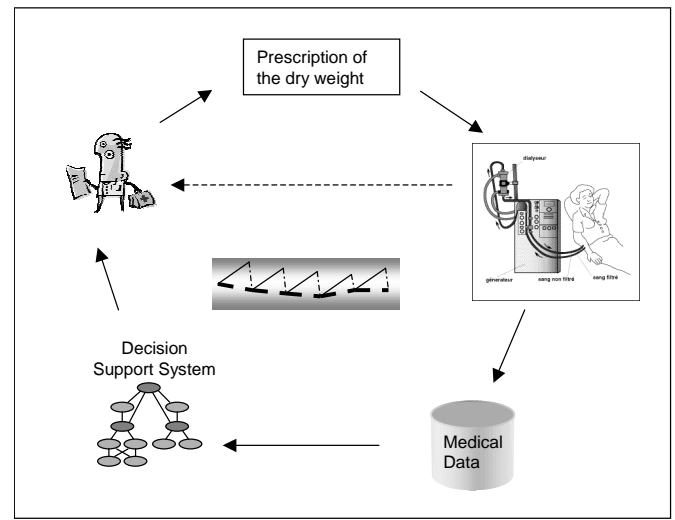

Figure 2. Information flows between the patient, the physician and the decision support system.

The purpose of our application is to monitor the dry weight of patients with chronic renal failure treated by hemodialysis, and to help the physician in his decision. It cannot replace the physician's expertise for making the decision. The responsibility of the treatment remains in the hands of the physician.

\section{A belief network model for dry weight ad- justment monitoring}

The first step in developing a decision support system is to define a sufficient set of indicators for making the diagnosis. This work was done with the collaboration of the nephrologists. We limited the data used for the diagnosis to elements which can easily be acquired without the presence of a physician since the system should be adapted to patients treated at autodialysis units. This excludes clinical signs such as edema ${ }^{1}$. Each indicator is associated to an observed variable in the Bayesian network.

- Blood pressure: The more the blood flow contains water the bigger its volume is and as a consequence

\footnotetext{
${ }^{1}$ swelling caused by excessive fluid in the body
} 
the force of the blood pushing against the walls of the arteries grows. High/low blood pressure can be a symptom of overhydration/dehydration. Blood pressure is measured at the beginning $(\mathrm{BPb})$ and at the end (BPa) of the dialysis. Orthostatic hypotension means low blood pressure on upright posture and it can be a symptom of dehydration. The difference between blood pressure on lying and upright posture is measured before $(\mathrm{OHb})$ and after $(\mathrm{OHa})$ the dialysis.

- Weight: Except in the case where one is starving or infected, real tissue weight is not gained or lost rapidly. If the patient's weight grows by one kilogram in one week, there is a risk that it is one kilogram of water. The evolution of the patient weight is a key element for dry weight diagnosis. The weight of the patient is measured before $(\mathrm{Wb})$ and after (Wa) the dialysis. At the end of the dialysis the weight of the patient should be nearly equal to the dry weight prescribed by the physician.

- Ultrafiltration: This quantity (UFT) is calculated as the difference between the weight at the beginning of the dialysis and the dry weight estimated by the physician. It represents the amount of water to be extracted during the dialysis. The extraction speed of the water depending on the duration of the dialysis session is known as the ultrafiltration rate (UFR). If the (UF) / (UFR) is high the dialysis session may not be well tolerated by the patient which could result in low blood pressure at the end of the session.

The estimation of the hydration and the dry weight adjustment of the patient is represented by the following hidden variables:

\section{- Dry Weight (DW)}

- Hydration before the dialysis session $(\mathrm{Hb})$

- Hydration after the dialysis session (Ha)

The theory of Bayesian networks allows us to represent relationships between these observed and hidden variables in a probabilistic way which is well adapted to the uncertainty inherent to medical questions. Causal links between the variables are represented on figure 3 .

Observations made during a single dialysis session are not sufficient to properly evaluate the dry weight adjustment. Experiments conducted using this simple layer Bayesian network showed that the diagnosis was highly instable and thus producing false alerts. In order to represent the influence of past events over the present state of the patient, it is necessary to extend this model into a dynamic Bayesian network. We consider the dry weight as a first order Markov process so as to integrate the time dimension of this variable. The physician's action on the dry weight (increase / decrease) should also be taken into account when considering the time dimension of the variable. The diagnosis of dry weight adjustment should be based on the present dialysis session observations, the previously made estimation of the dry weight adjustment, and the potential actions made by the physician (dot lined node on figure 4).

In our Bayesian network, nodes representing the hydration state of the patient $(\mathrm{Hb})$ and $(\mathrm{Ha})$ are not connected between successive layers (corresponding to successive dialysis sessions). This choice can be justified as follows: contrary to the dry weight, the hydration is highly sensible to daily variations depending for example on the patients meals, the temperature or the physical efforts made by the patient. The influence of these external causes makes a causal link between the hydration variables of successive sessions irrelevant.

Our Bayesian network is based on discrete variables. Its structure as well as its parameters are based on human expertise. They have been defined with the collaboration of nephrologists. The dynamic Bayesian network data input is pre-processed in order to convert the medical information into a probability distribution over the network variables. The observations made over the three past sessions are entered as evidences and propagated into the dynamic Bayesian network with the exact inference algorithm JLO [4]. Thus we get the a posteriori probability distributions over the hidden variables of the network.

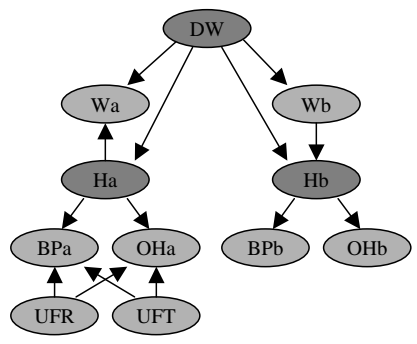

Figure 3. Simple layer Bayesian Network for dry weight adjustment diagnosis

\section{Preprocessing of medical data}

In order to be entered as soft-evidences into the dynamic Bayesian network, daily measures are transformed with fuzzy sigmoid operators into likelihoods to be greater than, equal to or lower than a given base. This preprocessing can be decomposed into three stages:

- Centering: The first stage consists in centering the measure around a reference value considered as nor- 


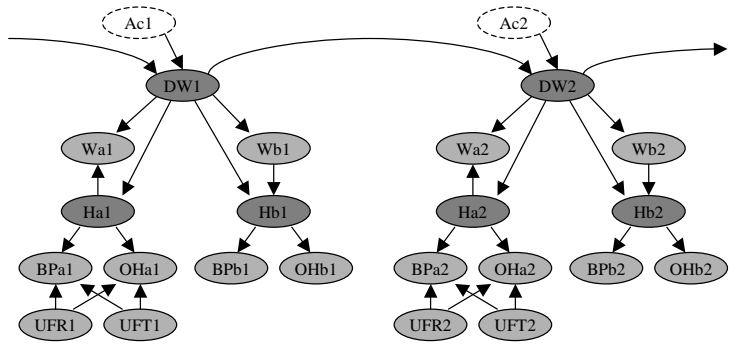

Figure 4. A Dynamic Bayesian Network model for dry weight adjustment diagnosis

mal for the patient. This reference is calculated based on statistics on the value's record history. For example the blood pressure is centered around the average blood pressure calculated for the patient over the last 15 days. The normal value for the weight at the end of the dialysis session (Wa) is the dry weight. This stage can be considered as an adaptation of the model input to the patient's data.

- Normalization: A tolerance is defined for each medical measure (weight, blood pressure, ultrafiltration) in order to normalize the values between -1 and 1 . This tolerance defines the width of the measure interval corresponding to the normal state of the discrete variable. When the value is saturated (equal to 1 or -1 ), it is a certainty that the variable is in state lower than or greater than the base.

- Discretization: The discretization consists in converting the normalized value into likelihoods. In our case, the variables of the Bayesian network have three states: low, normal, and high. The corresponding likelihoods are given by three fuzzy filters (see representation figure 5). The use of fuzzy filters has the advantage of

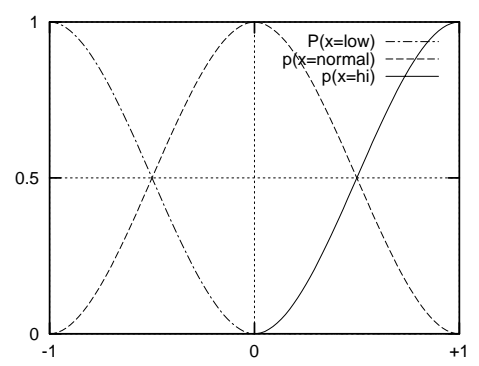

Figure 5. Probability density functions

preventing threshold effects.

\section{Interpretation of a posteriori probabilities}

The diagnosis produced by the Bayesian network is the a posteriori probability distribution of the hidden variable $D W$. The belief that the dry weight should be increased/decreased is given by the probability distribution $P(D W \mid$ observations $)$.

We used a simple threshold alarm for generating alerts. The system generates an alert when the probability $P(D W=$ high $)$ or $P(D W=$ low $)$ is greater than 0.5 for two consecutive sessions.

The physician has the possibility to consult the system diagnosis which is presented as a single curve for simplification reasons. This curve is obtained by subtracting $P(D W=l o w)$ from $P(D W=h i g h)$. This gives a representation between -1 and 1 indicating that the dry weight is low, normal or high. See figure 6 for an example of diagnostic curve.

\section{Experimental results}

In this section, we present the results of an experimentation conducted for more than a year in dialysis units. The dialysis sessions of about one hundred patients have been monitored by our decision support system.

Figure 6 shows an example of a diagnosis output over three months for a patient treated with hemodialysis. It shows the diagnosis and alerts compared to the physician's decisions. Each dot corresponds to an email sent to the physician alerting him of a high/low dry weight. Arrows correspond to sessions where the physician decided to increase or decrease the dry weight. Between 11/28/2004 and $01 / 23 / 2005$ several alerts were generated indicating that the dry weight was too high. These alerts were approved by the physician who indeed decreased the dry weight several times. On 02/25/2005 a low dry weight alert was generated followed by an increase of the dry weight by the physician.

Conclusions of nephrologists participating at the experimentation are the following. Primary results on care quality evolution are encouraging. The program gets the approbation of users not directly involved in the experimentation. Patients are satisfied to have access to their session data, they feel under better surveillance and are more respectful of medical prescriptions. The expert system is reliable and in agreement with medical decisions most of the time. It seems that some signals, directly given by the dialysis device (like the hemoscan which measures blood volume variations during the dialysis session), could help the expert system to anticipate the evolution of the dry weight. 


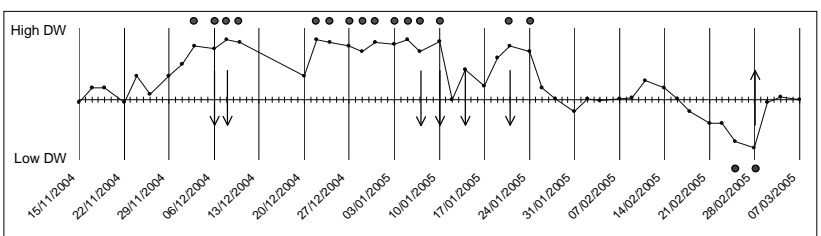

Figure 6. Monitoring of the dry weight adjustment of a patient treated by hemodialysis

\section{Conclusion}

In this paper, we described an application of dynamic Bayesian networks to the monitoring of patients treated by hemodialysis. The purpose of this decision support system is to help the physicians with the estimation of the dry weight.

The diagnosis as we presented it in this article is obtained from the patient data (weight, blood pressure...) as well as the data of the dialysis session (dry weight, duration...). A pre-treatment is used in order to transform medical data into standardized data usable by the system.

The analysis of care quality gives good results. Alerts generated by the expert system are reliable and its diagnosis is most of the time in agreement with the physician's decisions. Signals directly given by the dialysis device will be added to the expert system. It seems that some of these signals could help to anticipate the evolution of the dry weight.

In spite of the good results of our system, the estimation of the dry weight remains a rather complex problem even with the physicians' experience. The value of the dry weight is subjected to variations depending on the evolution of the patient's body fat mass. The indicators which permit the diagnosis of the dry weight (as for example blood pressure) are subjected to various external factors (meals, drugs...). All these external factors make the estimation of the dry weight very difficult.

Future work relates to the following points:

- The parameters used are generic, and therefore badly adapted to the particular cases, as for example heart failure patients. The generic model could be adapted to each patient by the definition of personalized profiles. These specific models would permit a finer diagnosis of the dry weight adjustment.

- The dialysis machine has several sensors which provide data throughout the dialysis session. The next development stage is to integrate some of them in the system analysis. We will focus on the data which gives information about the water volume in the organism like the hemoscan. The integration of such elements could help to anticipate the evolution of the dry weight.

\section{Acknowledgment}

This work was done in collaboration with physicians of the ALTIR (Association Lorraine pour le Traitement de l'insuffisance Rénale), the Diatelic company, and the Gambro company within the framework of INRIA (Institut National de Recherche en Informatique et en Automatique) action DialHemo.

\section{References}

[1] G. Cooper. Nestor: A computer-based medical diagnosis that integrates causal and probabilistic knowledge. Technical report, Stanford University, CA, 1984.

[2] J. Eugene Santos. On the generation of alternative explanations with implications for belief revision. In Proceedings of the seventh conference (1991) on Uncertainty in artificial intelligence, pages 339-347, San Francisco, CA, USA, 1991. Morgan Kaufmann Publishers Inc.

[3] M. Henrion. Towards efficient probabilistic diagnosis in multiply connected belief networks. In R. M. Oliver and J. Q. Smith, editors, Influence Diagrams, Belief Nets and Decision Analysis, pages 385-410. John Wiley and Sons, New York, NY, 1990.

[4] F. Jensen, S. Lauritzen, and K. Olsen. Bayesian updating in recursive graphical models by local computations. In Computational Statisticals Quarterly, 4:269-282, 1990.

[5] L. Lauritzen, G. Cowell, Robert, Dawid, A.Philip, S. , and D. J.Spiegelhalter. Probabilistic Networks and Expert Systems. Springer-Verlag New York, Inc, 1999.

[6] P. Murphy. Dynamic Bayesian Networks : Representation, Inference and Learning. $\mathrm{PhD}$ thesis, University of California Berkeley, 2002.

[7] Organisation. W.h. Technical report, http://www.who.int, 1997.

[8] J. Pearl. Fusion, propagation, and structuring in belief networks. Artif. Intell., 29(3):241-288, 1986.

[9] J. Pearl. Probabilistic reasoning in intelligent systems : Networks of plausible inference. Morgan Kaufman Publishers, Inc., San Mateo, CA, 2nd edition, 1988.

[10] L. R. Rabiner. A tutorial on hidden markov models and selected applications in speech recognition. pages 267-296, 1990. 